\title{
INTRAPULMONARY RUPTURE OF HYDATID CYSTS OF THE LIVER \\ BY
}

\author{
H. TOOLE, J. PROPATORIDIS, AND N. PANGALOS \\ From the Second Surgical University Clinic, Athens
}

(RECEIVED FOR PUBLICATION APRIL 18, 1953)

\begin{abstract}
Modern Greece is severely infested by hydatid disease. In two statistical studies made by one of us (Toole, 1930, 1937) 5,107 cases of hydatid cysts, observed in Greece between 1900 and 1936, have been collected. In a recent report Maccas and Kourias (1951) completed the statistics up to 1950 , adding another 6,000 cases. Seven hundred and sixteen cases were operated upon during 1949, an indication of the present frequency of hydatid disease in Greece. According to Maccas and Kourias the five countries most infested by echinococcus are, in the order of its incidence, Uruguay, Cyprus, Greece, Chile, and Argentina. Another interesting observation from Greek statistics is the frequency of lung echinococcus, amounting to $30 \%$. A high incidence of pulmonary cysts is also recorded in Cyprus, Italy, and Australia. It is not the aim of the present article to dwell on the frequency or the pathology of the cysts, but to describe the clinical aspects of a severe if rare pulmonary complication due to rupture of cysts of the liver.
\end{abstract}

\section{Pathology and Statistics}

For the following description we are to a great extent dependent on the publications of Dévé (1935) and Dew (1928). The expanding liver cyst, if not operated upon or in the rare event of the parasite dying, inevitably leads to rupture of the adventitia. Generally the cyst ruptures into the peritoneum causing secondary echinococcosis of the peritoneum or choloperitoneum. Next in frequency is rupture into the biliary passages, simulating gall-stone colic.

Cysts situated near the upper liver surface have a tendency to grow upwards, elevating the diaphragm, and uncomplicated liver cysts may make their way through the diaphragm and become intrathoracic. Usually, however, they rupture into the pleural cavity or into the bronchial tree.

Intrathoracic rupture is usually a sequel to suppuration in the cyst causing destruction of the pericyst and of the surrounding tissues. Suppur- $\rightarrow$ ation was present in $93 \%$ of the ruptured multi- $\frac{\text { O }}{5}$ vesicular and in $66 \%$ of the univesicular cysts $\rightarrow$ (Dévé, 1935). Another cause of intrathoracic rupture is exploratory puncture of the liver, $\overparen{Q}$ especially if followed by suppuration of the cyst. The resistance opposed by the diaphragm accounts $\mathbb{\Phi}$ for the rarity of intrathoracic rupture. Of 1,743 liver cysts collected by Dévé only $44(2.5 \%)$ ruptured into the thorax. The incidence of rupture was only $1 \%$ in 1,250 liver cysts observed in Greece. In Iceland the position appears to be different because out of 37 liver cysts 10 ruptured into the bronchi. Twenty-nine, however, of these cysts were calcified (Claessen, 1928). Dévé (1935) collected 380 ruptures into the thorax. Up to $1939 \vec{F}$ we were able to add another 14 cases, eight from the literature and six Greek cases. Dévé states that the relative frequency of rupture is in males? 152 and in females 111 cases. The highest incidence is at the age of $30-40$ years. In $21.6 \%$ of cases the cyst ruptured into the pleural cavity, in $\stackrel{x}{x}$ $74.7 \%$ into the bronchi, and in $3.6 \%$ in both directions.

Intrapleural rupture is a serious complication occurring as a rule suddenly and accompanied by severe pain, dyspnoea, cyanosis, and collapse. If not fatal, an empyema develops containing bile- $\rightarrow$ stained foetid pus and hydatid material. We shall not refer to intrapleural rupture as it is outside the scope of the present study.

Perforation of the diaphragm, as shown from five necropsies, occurred in four cases through the $\omega$ muscular part and in one through the tendinous? part. In some cases multiple perforations have been found. The opening in the diaphragm may attain the size of a crown, but usually it is much? smaller.


right liver lobe and in 11 it was situated in the left $\frac{?}{\square}$ lobe. Most of the cysts ruptured into the right $\varrho$ lower lobe. Only eight ruptured into the left lower? 


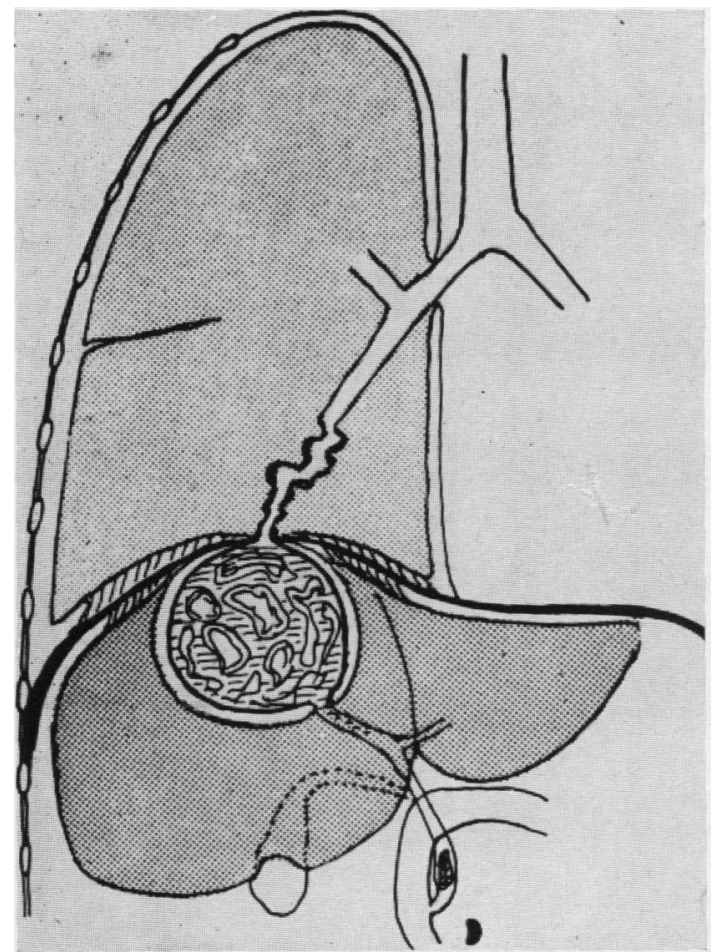

Fig. 1

lobe of the lung, but three ruptured into the right middle lobe. There are two types of rupture. In the first the suppurating liver cyst opened by a sinuous track through the adherent diaphragm and the lung into a bronchus (Fig. 1). In the second type an intrapulmonary cavity had formed; this was lined with necrotic tissue. This cavity communicated with a bronchus and contained pus, bile, and hydatid membrane (Fig. 2).

The presence of a cavity is recorded in $30 \%$ of 271 cases of rupture, but a cavity was found in $70 \%$ of the 83 necropsies performed. It seems probable that in many instances the cavity is not recognized clinically.

At operation or at necropsy a pulmonary cavity was occasionally found not communicating with a bronchus, as in our Case 5. This shows that cavity formation may often precede the rupture into the bronchus. There are variations of the above types. For instance, the rupture may occur through a subphrenic abscess or through an encysted basal empyema. The general pleural cavity may also become secondarily infected or the rupture may take place simultaneously into the bronchus and into the pleural cavity, or the intrapulmonary rupture may follow that of the pleural cavity. Not infrequently $(17-21 \%)$ there is a coexistent rupture into the bile ducts and this generally precedes the intrapulmonary rupture. Occasionally the common bile-duct is blocked by hydatid membrane so that the entire flow of bile is expectorated.

\section{Symptomatology}

Before rupture occurs symptoms are indefinite. Epigastric discomfort, cough from diaphragmatic irritation, haemoptysis, or moderate expectoration may be present. Hepatic or shoulder pain is more constant. Attacks of jaundice are mentioned in about one-third of the cases. Dévé attributed these attacks to rupture of a cyst into the bile passages, but in some cases they may have been due to the associated hepatitis or cholangitis. Sudden rupture occurred in $56 \%$ of the cases and was then accompanied by a sharp, tearing pain. Expectoration of frothy, blood-stained fluid, together with severe paroxysms of coughing and cyanosis followed. Death from suffocation or anaphylactic shock was mentioned in six cases.

The amount of expectoration may at first exceed 30-50 oz. In the following days expectoration may



Fig. 2 
become more or less bilious and foetid. Membranes are intermittently expectorated but in $20 \%$ of cases they are altogether missing. In $38 \%$ of ruptures the onset of symptoms was not sudden but gradually a purulent bile-stained expectoration developed. An even milder onset was mentioned in $6 \%$ of ruptures, followed by a course protracted over years (11 and 20 years according to Claessen).

If bile is mixed with sputum it is an important diagnostic sign. The amount of the expectorated bile varies and often its presence is intermittent. In $10 \%$ of cases no bile was found in the sputum during the whole course of the illness. In such cases biochemical tests might be of value for detecting bile in the sputum.

Clinical examination often reveals hepatomegaly and elevation of the diaphragm. In some cases, owing to the presence of gas in the cyst, a zone of tympanitic resonance may be detected. In a personal observation (Case 1) this symptom was caused by collapse of the superior liver surface following rupture of the liver cyst. In percussion the elevation of the diaphragm is often masked by dullness at the lung base or by pleural effusion. On auscultation there may be friction, rales, amphoric breathing, metallic tinkling, or a succussion splash.

Radiological examination often reveals elevation or distortion of the diaphragm and impaired respiratory movement. Sometimes a gas-containing liver cyst or a calcified one is seen inside the liver shadow. In the lung base a cavity containing fluid and gas is occasionally outlined. Exceptionally a spherical shadow simulating a simple lung cyst is seen (Kingreen, 1926 ; Ljubarski, 1926, case 5 ). In some cases with a protracted course the only indication of rupture is an opaque band in the radiographs leading to the hilus.

\section{BRONCHOGRAPHY}

Bronchography rarely revealed the site of the internal bronchial fistula. Retrograde bronchography through the opened liver cyst may give valuable information as in Case 10. The results of surgical treatment depend on accurate localization of the perforation in the diaphragm. It is also important to know before operation the place at which the collapsed liver cyst is more accessible from the surface of the body. It is equally important to verify the existence of any lung cavity and to localize it exactly. Bronchography, tomography, and bronchoscopy will therefore be of value.

Aspiration of bile-stained pus from the lung or the liver cavity remains the surest means of localizing the lesion, but owing to collapse of the cyst and blocking of the needle by membrane, exploratory puncture is negative in $25 \%$ of cases.

\section{Differential Diagnosis}

Differential diagnosis in countries infested with hydatid cyst is not difficult if bile is expectorated, because hepato-bronchial fistulae from other causes, excepting amoebic abscess of the liver, are rare. Morton and Philips up to 1928 collected only 47 cases of fistulae from different causes, not including the rupture of a hydatid cyst or of an amoebic abscess. Until 1935 the number of such cases amounted to 57 according to French (1935). Thirty-five cases had fistulae caused by cholelithiasis (Razemon, Bizard, and Lambret, 1933). In Greece, where amoebic dysentery is common, the rupture of an amoebic abscess must be taken into consideration. An absolute proof of the hydatid origin of intrabronchial rupture is the expectoration of hydatid material. If it is missing a positive intradermal reaction, complement fixation test, and eosinophil count are valuable in diagnosis. Unfortunately they are often negative, owing to the death of the parasite and to the lowered resistance of the patient. If no bile is expectorated the most common error is the diagnosis of a ruptured lung cyst.

\section{Radiological Examination}

Radiological examination may be of great help in diagnosis, although ruptured and collapsed lung cysts may elude detection in cases of so-called dry retention. In other cases the outlines of the ruptured lung cyst may be blurred by pericystic inflammation or by pleural effusion. Lastly the lung abscess which forms occasionally after rupture of the liver cyst, may simulate a ruptured lung cyst. By using modern radiological technique these errors will become less frequent. Diagnostic errors may also occur in cases of ruptured lung abscess, encysted empyema, suppurating non-parasitic lung cysts, and so forth. The ultimate prognosis of intrapulmonary rupture is very grave. A fatal outcome occurs in $50 \%$ of cases treated conservatively against $33 \%$ cured and $15 \%$ presumably cured. The causes of death in this series were lung abscess or gangrene, recurrent haemoptysis, rupture into the pleural cavity, cholangitis, peritonitis, pericarditis, thrombosis, and sepsis. In chronic cases residual lung cavities and bronchiectasis developed. In eight cases tuberculosis followed. Even patients considered as definitely cured may have a recurrence many years later as, for instance, a patient of Claessen who relapsed after 31 years. 


\section{TREATMENT}

Since the prospects of natural cure are remote and the risks of temporizing great, operative treatment is indicated. The operation must be performed before irreversible lesions have become established in the lung and the liver and before the resistance of the patient is lowered. In most cases resistance becomes low very soon in consequence of the severe inflammatory process in the affected organs and as a result of the incessant paroxysms of coughing and the profuse expectoration.

Operative mortality according to Dévé's (1935) statistics is high (39\%). These figures are based on older observations, but the advent of antibiotics and the progress in anaesthesia and resuscitation have improved the operative results.

In treating intrapulmonary rupture it is essential first to control the expectoration of bile and foetid material by evacuating and draining the liver cyst from the most accessible position. This is not always possible, owing to the thickness of the intervening liver parenchyma in deeply situated cysts. If daughter cysts are left behind they may cause a prolonged drainage and the recurrence of the hepatobronchial fistula. This happened in 18 cases cited by Dévé. Most of the ruptured liver cysts must be approached through the diaphragm after rib resection. Usually pleural adhesions are found, but if none are there the risk of infection in the pleural cavity must be faced. In cysts situated on the anterior surface of the liver the approach through the abdominal wall is preferable. Nario (1948) in a recent report maintains that simple drainage of the cyst cannot heal the bronchial fistula. The conclusion is far too general, even admitting the existence of such cases. Perusal of the literature has convinced us that most cases can be cured by simple drainage of the liver cyst or even after drainage of the secondary lung cavity or of the associated empyema. It is more reasonable therefore to follow Barrett (1947), who first drains the liver cyst, and then at a second stage performs a lobectomy if irreversible lesions have developed in the lobe. The experience of Greek surgeons suggests that such lesions develop only in very chronic cases. We believe that special conditions may have induced Armand Ugon (1950) to perform eight lobectomies out of a total of 10 operations for intrapulmonary rupture.

It is often difficult to localize the site of the liver cyst. In this case the hepatobronchial communication must be severed either by liberating the adherent lung base from the diaphragm or by dividing the adhesions between the diaphragm and the superior liver surface. The risk of such extensive operations must not be underestimated.

Post-operatively care must be taken to drain the liver cyst adequately as long as the flow of bile continues to prevent the recurrence of expectoration of bile. A prolonged flow is likely to occur in cysts with thick or calcareous adventitia. In these cases the cyst wall may exfoliate and be discharged. Secondary haemorrhage may occur during the separation of adventitia. If the stool becomes clay-coloured a block in the common bile-duct may be the cause. Usually in such cases following simple drainage of the cyst the duct unblocks spontaneously. In gross obstruction drainage of the common bile-duct may become necessary to avert the threat of cholangitis. This additional intervention carries with it a great risk owing to the grave condition of the patient.

\section{Case Reports}

CASE 1.-A woman, aged 48, five months before admission was feverish for a month. Later she had violent attacks of coughing and suffocation, accompanied by the expectoration of frothy, bile-stained fluid. Then followed an interval of relative amelioration, when no bile was expectorated. Afterwards the same symptoms recurred repeatedly. She was admitted to hospital in February, 1932. She was in a poor general condition, but the diaphragm was not elevated. Posteriorly a tympanitic area the size of the palm of the hand was found in the hepatic region. The patient expectorated about $30 \mathrm{oz}$. of sputum daily. The intradermal reaction was strongly positive, complement fixation tests were positive, and an exploratory puncture was negative.

The ninth and tenth ribs were dissected and diffuse pleural adhesions found. The diaphragm was incised, but the liver cyst could not be traced. The diaphragm was sutured and the adherent lung freed. Near the cupola an egg-sized liver cyst was opened containing bile and collapsed daughter cysts. Drainage was carried out.

At first there was a profuse flow of bile from the wound. The sputum was twice transiently bile-stained. However, the patient improved rapidly and left hospital after a month.

CASE 2.-A man, 27 years old, was operated upon 50 days before admission for a cyst of the superior liver surface. The wound was sutured but the cavity was drained later because bile was leaking. Three weeks later after paroxysms of coughing he expectorated bile-stained fluid. His temperature was $39^{\circ} \mathrm{C}$. for 10 days. Thereafter expectoration became less profuse and was only intermittently bile-stained. His temperature fell to $38^{\circ} \mathrm{C}$. On admission dullness reaching to the scapula was elicited, and radiography showed elevation of the diaphragm and a diffuse opacity in the lung base. The intradermal reaction was strongly 
positive. Pus was aspirated by puncture beneath the angle of the scapula.

At operation in February, 1932, the seventh rib was resected. The pleural space was adherent. An orange-sized cavity in the lung was opened; it was lined with necrotic tissue and contained bile-stained membrane. From the perforation in the diaphragm pure bile flowed. Drainage was performed.

After operation the bile-stained expectoration ceased, but the wound discharged bile and later pus in diminishing quantities. The patient was discharged after a month.

CAse 3.-A woman, aged 20, 20 days before admission felt unwell. After two days she developed a pain in the right hypochondrium and a high temperature $\left(40^{\circ} \mathrm{C}\right.$.). Three days later she expectorated bilestained fluid, which, in the following days, became purulent. The high temperature persisted $\left(38-40^{\circ} \mathrm{C}\right.$.). Upon admission liver dullness reached 2 in. below the angle of the scapula, and the daily quantity of sputum was $8 \mathrm{oz}$. The intradermal reaction was positive. Pus was aspirated from the ninth intercostal space.

At operation in May, 1932, the tenth rib was resected. Pleural adhesions were found. The diaphragm was incised and a small collection of pus opened. From thence a sinuous tract leading to a voluminous hepatic cyst situated ventrally was found.

The temperature became normal after five days, and expectoration ceased. The flow of bile from the wound diminished progressively. The patient was discharged after 20 days.

CASE 4.-A man, 21 years old, seven months before admission felt a severe attack of pain in the right hypochondrium which lasted for 12 hours. After five days he experienced a second attack of pain accompanied by fever $\left(39^{\circ} \mathrm{C}\right.$.). A few days later he became jaundiced and remained so for a month. Then he experienced a colicky pain, followed by persistent pyrexia. A month later, during violent paroxysms of coughing, he developed profuse, bile-stained expectoration. $\mathrm{He}$ was twice operated upon without result. The high temperature $\left(38.5^{\circ} \mathrm{C}\right.$.) persisted for two months. Intermittently he expectorated membranes. Twenty days before admission he had a haemoptysis.

On admission dullness reached to the fourth rib posteriorly. Radiography showed a diffuse shadow from the fourth rib downwards. Punctures were negative.

At operation in March, 1933, an incision was made on the old scar over the eighth rib. The adherent lung base was freed. Near the cupola a voluminous liver cyst, containing pus and disintegrated daughter cysts, was opened and drainage established. The patient did not rally and died next day.

CASE 5.-A man, 23 years old, one and a half months before had complained of discomfort. After 10 days with a high temperature $\left(39^{\circ} \mathrm{C}\right.$.) accompanied by pain in the right axilla he was admitted to hospital. On admission a palm-sized dullness was found beneath the scapular angle. Radiography revealed a spherical shadow bordering on the diaphragm. The intradermal reaction was negative.

At operation in May, 1933, the ninth rib was resected. The lung was fixed by sutures to the chest wall, and pus from the middle lobe was aspirated by puncture. The cyst was evacuated and drained. Bilestained pus and membrane were profusely discharged for a week. Each time the tube became blocked expectoration became bile-stained. Thereafter improvement was rapid. The patient was discharged after 26 days.

Case 6.-A woman, aged 50, suffered from cough for four months before admission. Twice she expectorated small quantities of bile-stained fluid. A month before admission she expectorated $60 \mathrm{oz}$. of clear liquid. Four days later she coughed up a daughter cyst. After that she expectorated bile-stained fluid and membrane. On admission she was jaundiced. The diaphragm was not elevated. The liver border was palpated four fingerbreadths below the costal margin. She was expectorating $15-30 \mathrm{oz}$. of bilestained fluid daily, and had incessant paroxysms of coughing. Radiography revealed a slight opacity of the right lung base. The intradermal reaction was positive and punctures for pus were negative.

At operation in April, 1939, an incision was made parallel to the costal margin. When a hard mass near the cupola was punctured bile-stained liquid escaped. The costal arch was mobilized by section of the rib cartilages. A thick-walled liver cavity the size of an egg, but not containing hydatid elements, was opened and drained. After operation bile appeared intermittently in the sputum owing to inefficient drainage. After one month the wound was discharging frank pus. The patient left hospital a month later.

CASE 7.-A man, 35 years old, was operated on three months before admission for a large suppurating subdiaphragmatic cyst. Bile flowed profusely from the wound, and a bile fistula developed. At intervals he had a high fever caused by retention of pus. Two weeks before admission he had a high temperature and paroxysms of coughing, and after two days abundant bile-stained expectoration. Although the external fistula was again discharging bile, bilious expectorations continued.

On admission the right diaphragm was found to be $\mathrm{N}$ moderately elevated, and was confirmed by radio- $\sigma$ graphy which also revealed a blurred shadow over $N$ the right lung base.

At operation in May, 1945, the seventh and eighth ribs were resected. The pleural space was adherent. The diaphragm was incised along the fistula, which was leading to a liver cavity under the cupola. Tamponade was carried out.

After operation abundant bile flowed from the $\tau$ wound for over a month, and occasional expectoration was bile-stained during the first two weeks. The $\mathbb{D}$ patient was discharged after two and a half months with a drained wound. Six months later a sinus still persisted, intermittently discharging bile. 
CASE 8.-A man, 52 years old, 13 years before had experienced a colicky pain in the right hypochondrium. After two years he was feverish and jaundiced for 20 days. This episode was repeated four years later. He suffered occasionally from colicky pains. For six months he complained of persistent pain accompanied by pyrexia and rigors. A liver biopsy confirmed the diagnosis of cholangitis. A month before admission bile-stained fluid was withdrawn by puncture from the right chest. Culture revealed pneumonococcus.

On admission he had a high temperature (38$39^{\circ} \mathrm{C}$.) and dullness over the right lung base. He was producing about $8 \mathrm{oz}$. of sputum daily. Tubercle bacilli were not found. Radiography revealed a right pyopneumothorax with different fluid levels.

At operation in March, 1947, the ninth rib was resected. The pleural space was adherent and pus was found at liver puncture. A small abscess was opened and then an empty cavity of moderate size. Drainage was performed.

Twelve days after operation another liver abscess was opened through the wound. A week later a voluminous hydatid cyst was discharged with the drainage. After 10 days a purulent intrapleural effusion was drained. The patient then improved rapidly and was discharged 26 days after the last operation.

$\mathrm{He}$ was admitted again after five months because two weeks before he became feverish and expectorated bile-stained fluid. Puncture at the site of the former operation revealed an air-filled cavity, but an attempt to drain it failed. After two weeks the lung base was freed from the adherent diaphragm and the hepato-bronchial fistula divided.

After operation the sputum was bile-stained from time to time, and bile flowed copiously from the wound. The stools were clay coloured, suggesting a block of the common bile duct. During the next two months the common bile-duct did not spontaneously unblock, and an attack of cholangitis followed. After the acute stage the common bile-duct was freed from the obstructing membrane and drained. The patient succumbed on the next day.

The following cases were operated upon at the Clinic of Social Insurance by Dr. K. Zacharakis and one of the present authors (N.P.).

CASE 9.-A man, 35 years old, three months before had been operated upon for three hepatic cysts. Twenty days before admission he became feverish. Two weeks later another suppurating liver cyst ruptured into the drained wound. On admission pus and collapsed daughter cysts were discharging profusely from the wound. The temperature was about $38.5^{\circ} \mathrm{C}$. A transient amelioration followed. Then the patient was again feverish for 20 days owing to an inflammatory process in the right lung base.

Radiography revealed elevation of the right diaphragm and a blurred shadow over the lung base. After a few days he became jaundiced. A painful mass was then palpated in the right hypochondrium.



FIG. 3.-Radiograph of Case 9 showing an interlobar effusion.

At operation in June, 1951, an abdominal incision was made and a voluminous suppurating liver cyst was drained.

Twenty days after operation the patient had again a high temperature, persistent cough, and jaundice. Two months after operation he felt a stabbing pain in the chest followed by bile-stained expectoration, amounting to $16 \mathrm{oz}$. Radiography showed, in addition to the former findings, an interlobar effusion (Fig. 3). The patient succumbed two months later from repeated haemoptysis.

CASE 10.-A woman, aged 23, nine months before had been operated upon for a hydatid cyst of the

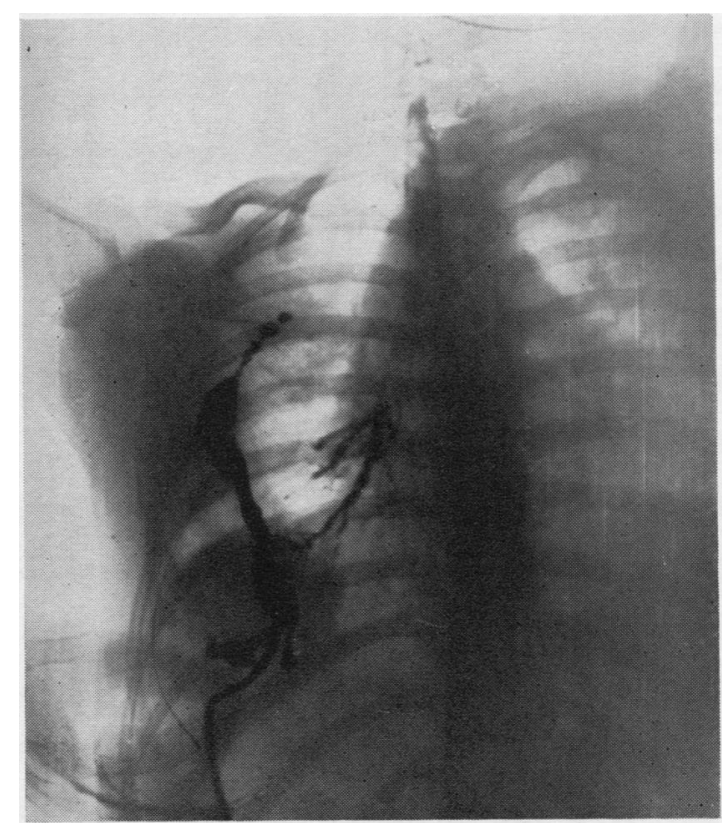

FIG. 4.-Radiograph of Case 10 showing a small liver cavity communicating with the bronchi of the inferior lobe of the lung. 
right lung. One month before admission she became feverish. On admission in September, 1951, she had high fever $\left(40^{\circ} \mathrm{C}\right.$.), dyspnoea, and jaundice. At percussion dullness was heard over the right hemithorax, and radiology revealed the presence of free fluid. In the pleural cavity a purulent fluid was aspirated, and the empyema was drained. The patient left hospital after three months.

Five months later she had a paroxysm of coughing, and expectorated bile-stained sputum. On the next day the wound opened and discharged pure bile. After that the sputum was no longer bile-stained, but when the patient was lying down the sputum tasted bitter. A radiograph taken after the injection of an opaque fluid into the sinus revealed the persistence of a small liver cavity communicating with the bronchi of the inferior lobe of the lung (Fig. 4). The sinus closed after a week. The patient was followed up for a month, during which she had no recurrence.

CASE 11.-A man, aged 56, two weeks before admission became feverish and complained of mild pain in the right hypochondrium. On admission a rounded, movable tumour was palpated under the right costal arch and a lesser one beneath the xiphoid. Radiography revealed an elevation of the right diaphragm and a diffuse shadow in the contiguous lung base.

At operation in August, 1952, two hydatid cysts, each the size of a mandarin orange, of the mesocolon and of the liver margin were extirpated. A third voluminous cyst in the superior surface of the liver was drained. Ten days after operation the temperature rose to $40^{\circ} \mathrm{C}$., and a week later another suppur-



FIG. 5.-Radiograph of Case 11 showing a hepatic cavity. ated liver cyst ruptured into the wound. Following this an inflammatory process developed in the right lung base. The amount of sputum expectorated daily gradually rose to 10 to $18 \mathrm{oz}$., and finally became bilestained. The patient was at the same time jaundiced. The sitting posture reduced expectoration and after three days in this position bile disappeared from the sputum. A radiograph after the injection of an opaque fluid revealed a voluminous, partially collapsed hepatic cavity no longer communicating with the bronchi (Fig. 5). The patient gradually improved and will soon leave hospital.

Besides the cases described above we have treated two more of hepato-bronchial fistula, but the hydatid origin of these cases is not certain.

\section{SUMMARY}

Hepato-bronchial fistula is an infrequent though severe complication of hydatid liver cysts. In the majority of cases the rupture into the bronchus follows the suppuration of a cyst. Except for amoebic liver abscess, all other causes of hepatobronchial fistula are rare. Diagnosis is certain if hydatid elements are found in bile-stained expectoration. In the absence of these elements biological tests for hydatid disease may be of diagnostic value. If bile is missing from the sputum the rupture is generally ascribed to a lung cyst. Clinical and radiological examination rarely reveal the site of the ruptured liver cyst. Radiography may visualize the lung cavity formed secondarily after rupture of the liver cyst. Perforation usually takes place into the right lower lobe, seldom into the middle lobe or into the left lower lobe. It is important to localize the site of the ruptured liver cyst, and is effected in most instances by exploratory punctures. Puncture is, however, negative in about $25 \%$ of cases. Bronchography perhaps might be able to remedy this deficiency.

The prospects of natural cure are remote and the mortality is high in intrapulmonary rupture, therefore operative treatment is indicated. Operation must be performed before irreversible lesions develop and before the resistance of the patient is lowered. The mortality after operative treatment is high, but operative results have greatly improved of late. In treating intrapulmonary rupture it is essential to intercept the expectoration of bile and foetid material. This can be done by draining the ruptured cyst, if possible, from the most dependent position. Simple drainage will cure most cases of bronchial fistula. If it is impossible to localize the cyst before intervention, the hepato-bronchial communication must be severed, either by freeing the adherent lung base or by dividing the adhesions over the superior liver 
surface. The lesions of the affected lobe of the lung are irreversible only in the case of chronic intrapulmonary rupture, in which case lobectomy may become necessary. An associated obstruction of the common bile-duct by membrane is a very serious complication of intrathoracic rupture, especially if the duct does not unblock spontaneously.

Only early diagnosis and surgical treatment of the uncomplicated hydatid cyst of the liver can prevent the occurrence of the various complications to which the growing cyst leads invariably.

\section{Bibliography}

Armand Ugon, C. V. (1950). Arch. Chir. del Torace, 5, 85. (1951). Arch. urug. Med., 38, 201.

Barrett, N. R. (1947). Thorax, 2, 21.

Claessen, G. (1928). Acta radiol. Stockh., Suppl. 6.
Dévé, F. (1935). L'ouverture des kystes hydatiques du foie dans le poumon et les bronches. Ve Cong. Ann. Fed. Scienc. Med. Alg. Tun. Maroc Oran 10-13 April, 1935. With review of the literature.

Dew, H. R. (1928). Hydatid Disease. The Australasian Medical Publishing Co., Sydney.

Fogliati, A., and Bruzzone, P. (1952). Arch. Sci. med., 177, 209.

French, R. W. (1935). Arch. Surg., 30, 635.

Kingreen, O. (1926). Dtsch. Z. Chir., 197, 83.

Ljubarski, B. (1926). Arch. klin. Chir., 143, 718.

Maccas, M. (1951). Echinococcus must be stamped out in Greece. Published by the Greek Red Cross, Athens.

and Kourias, B. (1951). Arch. intern. Hidat., 12, 1-2, 61.

Merino, R. A. (1949). Acad. Peruana, 3, 143.

Morton, I., and Philips, E. W. (1928). Arch. Surg., 16, 697.

Nario, C. V. (1948). Pren. med. argent., 35, 1282.

Razemon, P., Bizard, G., and Lambret, M. (1933). Rev. Chir., Paris, 52, 485.

Sauvage, R. (1947). Mém. Acad. Chir., Paris, 73, 600.

Suárez, H., and Victorica, A. (1950). Arch. urug. Med., 37, 565.

Toole, H. (1930). Arch. klin. Chir., 159, 124.

(1932). Ibid., 172, 106.

- (1937). Ibid., 183, 459. 